\title{
MAGDALENA ZDRADA-COK
}

Université de Silésie à Katowice

iD 0000-0002-4777-4041

\section{Clichés orientalistes et stéréotypes formels dans un roman satirique : le cas de La vieille dame du riad de Fouad Laroui}

\author{
Orientalist cliches and formal stereotypes in the satirical novel \\ La vieille dame du riad by Fouad Laroui
}

\begin{abstract}
This article analyses the novel "La vieille dame du riad" (2011) by Fouad Laroui, a Moroccan-Dutch writer of French expression. We examine the strategies (generic and intertextual) used by the novelist to ridicule the Orientalist prejudices which persist in the current time. Generic hybridity (the presence of some elements of the detective story, fantasy novel and oriental tale) is used to develop the satirical dimension of the novel.
\end{abstract}

KeY Words : stereotype, generic hybridity, Orientalism, satire

Si dans le domaine de la psychologie sociale et surtout dans les études interculturelles, le terme de stéréotype renvoie à des images schématiques, rudimentaires, simplistes qui servent à caractériser une culture ou un groupe humain donnés, il ne possède pas de connotations aussi fermement péjoratives dans les études littéraires. Dans la théorie des genres et dans la pragmatique du texte littéraire, par-delà son acception péjorative qu'on ne saurait certes ignorer, le stéréotype fonctionne aussi comme une catégorie neutre, en tant que «socle de compétence qui permet à un lecteur non seulement de comprendre et d'interpréter, mais aussi de modaliser et d'évaluer un texte» (DufaYs, 1994: 80). Michel Riffaterre considère notamment le stéréotype comme "garant de la lisibilité et du sens du texte » (RIFFATERRE, 1979: 19), la stéréotypie permettant au texte de s'inscrire dans des schémas reconnaissables et dans un contexte socio-culturel donné.

Comme l'a démontré Jean-Louis Dufays, le stéréotype peut se présenter dans le texte littéraire sur trois plans: idéologique (c'est-à-dire au niveau des valeurs), 
thématico-narratif (actions, personnages, décors, etc.) et linguistique (norme langagière, syntagme, phrase, etc.) (DufaYs, 1994 : 77). Cette triple présence du stéréotype dans la littérature renvoie à trois catégories dont parle Anne Herschberg Pierrot: elocutio (on y retrouve des clichés et autres stéréotypes langagiers), dispositio (ce sont des poncifs en tant que thèmes littéraires) inventio (catégorie qui englobe les lieux communs) (Herschberg Pierrot, 1980: 338).

$\mathrm{Vu}$ ces acceptions nombreuses du terme, dans La vieille dame du riad, septième roman de Fouad Laroui publié en 2011, le stéréotype m'intéresse surtout du point de vue des études interculturelles et postcoloniales (et mon point de départ est l'orientalisme dans la perspective d'Edward W. Said). Mais j'analyse aussi la stéréotypie, en prenant en considération les aspects génériques et intertextuels du texte. Et ces deux approches restent bien-sûr intrinsèques, dans la mesure où le roman de Laroui en dénonçant les préjugés, joue - sur le plan de dispositio et d'elocutio avec plusieurs paradigmes formels banalisés. Ce qui m'intéresse donc, c'est un certain paradoxe: Laroui dénonce le stéréotype culturel et - dans ce but - s'en sert sur le plan formel.

La vieille dame du riad critique surtout les stéréotypes orientalistes qui perdurent en France à l'époque actuelle (l'histoire se passe en 2009-2010). Je rappelle à ce propos que dans son fameux essai, Edward W. Said définit l'orientalisme comme «un style occidental de domination, de restructuration et d'autorité sur l'Orient» (SAID, 2005 : 15). Said montre comment le concept de l'Orient a permis à l'Europe de se définir par contraste, comment l'Occident s'est renforcé et a précisé son identité en se démarquant d'un Orient qu'il prenait comme une forme inférieure et refoulée de lui-même. Cette thèse lui permet de démanteler le mécanisme de la stéréotypie dans la perception de l'Orient, et montrer comment l'Occident a renfermé l'Orient dans l'image de L'Autre.

Laroui reprend ce clivage dans l'histoire satirique des Girard, un couple de Parisiens qui décident d'acheter un riad au Maroc. En ignorant tout de l'histoire et de la réalité de ce pays, ils ne se contentent au départ de leur histoire que d'une poignée de généralisations et d'idées fausses. "On sera bientôt plus marocains que Bourguiba» (LAROUI, 2011: 56) ou encore «Tu es en plein désert, enfin dans une palmeraie, une sorte d'oasis et en même temps tu vois au loin la neige ...les neiges éternelles ! - Celles de Kilimadjaro ?» (LARouI, 2011 : 14); de telles phrases avec lesquelles les protagonistes se représentent Marrakech avant de s'y installer en disent long à la foi sur leur savoir et sur l'humour qui caractérise le roman.

En confrontant Cécile et François avec le personnage autant étrange qu'étranger de la vieille femme qu'ils découvrent dans la chambre du fond de leur riad nouvellement acquis, Fouad Laroui joue avec brio avec des concepts culturels bien ancrés dans la réflexion postcoloniale tels que l'altérité et l'étrangeté. Il représente, de manière certes caricaturale, comment certains occidentaux voient le monde ex-colonisé. En faisant des différences culturelles entre les 
Français et les Marocains l'objet de la satire, Laroui dénonce les préjugés qui caractérisent le regard qu'on porte sur l'Autre. S'il fait rire le lecteur, c'est surtout pour le faire réfléchir sur l'importance de l'histoire dans le monde actuel ainsi que sur les conséquences de l'ignorance qui semble se propager dans les sociétés occidentales.

Sur le plan rhétorique et narratif, cette vocation polémique du texte repose sur le recours à des schémas littéraires bien connus. Sa forme s'appuie sur des paradigmes formels conventionnels, utilisés au deuxième degré tels que roman comique, récit fantastique, roman policier et conte oral. Ils sont tous subordonnés à l'ironie qui fait loi dans le roman.

Le jeu avec le genre fantastique traditionnel concerne la représentation de l'héroïne éponyme. Assise dans la chambre du fond, pareille à une ombre, elle se dématérialise, devient de plus en plus pâle, s'efface presque dès que les autres s'approchent d'elle. Sa présence spectrale fait peur. Son statut ontologique génère le doute de celui qui la voit; son existence ambiguë n'est peut-être qu'un effet d'hallucination de celui qui regarde :

Il (le commissaire Chaâbane) jeta un regard halluciné sur la vieille dame. Elle semblait être une apparition maintenant. C'est à peine si on la distinguait, ombre sur ombre, dans le noir. Chaâbane hurla. [...]

- Mais qu'est-ce qui se passe?

- Je n'en sais rien. On nage dans une histoire des fous.

LAROUI, $20011: 76$

De toute évidence, Laroui se réfère à la convention classique de la représentation fantastique qui s'appuie - comme l'a expliqué Tzvetan Todorov - sur l'hésitation qu'éprouve le lecteur entre deux explications du phénomène: rationnelle (l'idée du trouble mental de celui qui regarde) et surnaturelle (on admet que l'insolite fait son entrée dans le monde ordinaire). D'après la définition présentée par Todorov dans L'Introduction à la littérature fantastique, «le fantastique, c'est l'hésitation éprouvée par un être qui ne connaît que les lois naturelles, face à un événement en apparence surnaturel» (Todorov, 1970: 29). Celui-ci, d'habitude maléfique, sème le trouble, menace le héros, déstabilise l'ordre du quotidien. C'est visible notamment dans l'épisode où la vieille dame, comme une Vénus d'Ille, «punit» pour ainsi dire le commissaire Chaâbane, personnage borné et despotique. À la fin, on le retrouve détruit par son expérience insolite :

[II] passait désormais ses journées dans un état d'hébétude pitoyable dans un coin de la Koutoubia, justement assis sur une natte, les yeux dans le vide. Peutêtre priait-il pour le salut de son âme, s'il en avait une. 
Le fantastique, avec l'effet de suspense et l'atmosphère de mystère qui lui sont propres, relève, chez Laroui, d'un jeu parodique et - placé au deuxième degré - constitue une source du comique.

Il en est de même avec le schéma du récit policier. Ce genre, surtout dans sa variante du roman à énigme (Todorov, 1971: 14) attire le lecteur par son caractère ludique (cf. Poslaniec, Houyel, 2001: 31): il l'invite à poser des hypothèses, coproduire des significations de l'intrigue, mener l'enquête afin de résoudre l'énigme. Dans le cas de La vieille dame du riad l'interprétation dite policière de l'histoire est soumise aux lois de la parodie et - par conséquent elle fait surtout rire le lecteur. Mais dans ce mélange de genres et conventions, le recours au schéma policier a également pour but de contrebalancer l'insolite et le surnaturel par le rationnel pour assurer une cohérence à l'histoire et dynamiser l'intrigue qui, somme toute, est prévisible et conventionnelle. Car la présence de la vieille dame au riad serait tout simplement - dans la perspective dite policière - le résultat du complot monté par le samsar Hmoudane, le voisin Mansour Abarro et le commissaire Chaâbane :

Tout de même, ce n'est pas une centenaire qui va nous chasser de chez nous. D'ailleurs, maintenant que j'y pense, c'est peut-être ça, le but de la manœuvre : ils nous vendent une maison, puis ils y plantent une complice grimée en spectre et ils attendent qu'on déguerpisse, morts de trouille.

LAROUI, $2011: 77$

D'ailleurs cette version des faits gagne en crédibilité à la fin de l'histoire quand les héros apprennent que l'ancien propriétaire du riad haj Fatmi est en fait l'arrière-grand-oncle de Mansour Abarro. Ce dernier aurait donc des motifs familiaux et matériels pour tromper les Girard et reprendre le riad.

Or, personnage fantastique, clou de l'intrigue policière, la vieille dame est avant tout une conteuse et un personnage du conte. Par rapport au conte oriental (Chelebourg, 2006: 79-88), les catégories fantastiques et policières apparaissent dans toute leur modestie, comme secondaires, ne servant en fin de compte qu'à assurer au récit une cohésion et une dimension comique. Le genre oral y possède des modalités beaucoup plus importantes. Le conte, c'est Mansour Abarro, le voisin déjà mentionné, professeur d'histoire à l'université qui le transmet à Cécile et François sous forme d'un manuscrit. Il prétend l'avoir appris de la bouche de la vieille dame, apparemment muette. Par la suite, lu par les Girard, le conte qui leur a été pour ainsi dire «confié» par Abarro constituera la deuxième partie (et centrale, parce que la structure du roman est tripartite). Elle s'intitule «Histoire de Tayeb» et est symbolique dans la mesure où c'est la vieille dame elle-même, condamnée jusqu'alors au silence qui assume la parole (Abarro n'étant qu'un simple scribe). Son message est très significatif. Elle s'avère être un symbole de la soumission et une figure subalterne pour plusieurs raisons: en tant que femme, en tant que noire, en tant qu'esclave et en tant que colonisée. 
De cette manière, elle symbolise la dignité du Maroc soumis au colonisateur. De plus, en tant que personnification du pays, elle illustre sa diversité culturelle et sa pluralité. D'autant plus qu'au fur et à mesure qu'on découvre l'histoire de Massouda, on se rend compte de son statut particulier au riad de haj Fatmi: esclave, elle incarne la dignité et la solidarité des opprimés.

Il faut que je précise aussi que le conte de la vieille dame Massouda met en abyme d'abord l'histoire de la colonisation du Maroc, la guerre du Rif et ensuite la lutte des tirailleurs marocains sur les fronts de la seconde guerre mondiale (la bataille de Monte Cassino y devient emblématique).

Nous voyons donc qu'à ce niveau du conte, on passe donc du stéréotype à l'archétype. Tayeb, fils de haj Fatmi devient l'archétype du Marocain qui se révolte contre le colonisateur et qui est pris ensuite dans l'engrenage de l'histoire. Son conte, maintenu dans un style élevé, volontairement didactique, propre à la légende nationale, montre surtout la complexité de l'identité marocaine, due à sa pluralité autant culturelle (arabe, berbère, juive) que linguistique. La dimension historique et culturelle du message est d'ailleurs explicite. Voilà ce que dit Cécile (qui a bien compris la leçon d'histoire) à propos de Tayeb: «C'est d'ailleurs moins un être de chair et de sang qu'un symbole : avec ses trois mères, la Berbère, l'Arabe et la Noire - il ne manque que la Juive - c'est le Marocain, l'archétype, le maître étalon » (LAROUI, $2011: 210$ ).

Par la suite le conte sera poursuivi et terminé par Cécile. Ce dénouement prend forme de l'avant-dernier chapitre du roman intitulé «La fin de Tayeb». De cette manière la boucle est bouclée parce que la Française s'est promis d'écrire un roman sur le Maroc. Et l'histoire ainsi racontée en est un. D'autant plus que Cécile en avait déjà proposé une première ébauche, rédigée à la hâte, à son arrivée au riad, juste avant de connaître la vieille dame. Ce texte initial était une ouverture, toute ratée, maintenue dans un style prétentieux et pleine de couleur orientaliste. Il n'est pas difficile de voir qu'en faisant écrire à Cécile la conclusion au conte de la vieille dame, Laroui dote son roman d'une certaine dimension didactique: Cécile a finalement la chance de se réhabiliter aux yeux du lecteur.

Il est intéressant de voir que le conte que se partagent les personnages (la Vieille dame emblématique et ses porte-parole successifs: Mansour Abarro et Cécile), par sa fonctionnalité symbolique, historique, mythique même, se ressource dans la tradition orientale dont s'inspiraient notamment les philosophes des Lumières (par exemple Montesquieu racontant les contes philosophiques et politiques dans Les Lettres persanes). Ainsi, le conte-parabole, le conte-message devient surtout pour Laroui un code discursif dont le but est d'illustrer une idée donnée et de faire réfléchir le lecteur. En même temps, Fouad Laroui inscrit son histoire dans la tradition propre au roman maghrébin francophone dans lequel l'oralité est la source de la polyphonie des discours, de la pluralité des voix et de l'hybridité des formes (cf. ZeKRI, 2006 : 78-118. 
En effet, nous retrouvons dans La vieille dame du riad des références intertextuelles à L'Enfant de sable de Tahar Ben Jelloun, le plus célèbre roman marocain francophone construit sous forme d'une série infinie de contes. Rappelons que dans ce roman, Ben Jelloun actualise Les Mille et Une Nuits tout en rendant explicitement hommage à Jorge Luis Borges, grand admirateur de la polyphonie inhérente à ce monument de la littérature orale arabe. Ben Jelloun tisse dans son roman une série de relations intertextuelles aux contes arabes et à Fictions de Borges (L'Aleph, Le livre de sable, etc.) (cf. GonTARD, 1993: 99-119). Il partage surtout l'admiration qu'éprouve Borges pour le caractère infini et ouvert des nuits arabes (BORGES, 1985 : 58-65).

A son tour Laroui, en reprenant la tradition propre au conte oriental, utilise les stratégies discursives célébrées - pour ainsi dire - par L'Enfant de sable. Ainsi, comme le premier conteur de Ben Jelloun, Mansour frustre son auditoire : il commence à raconter, pour abandonner l'histoire inachevée; il accentue la curiosité de son public. Je cite quelques réactions de Cécile et François impatients: «Il nous fait le coup de Shéhérazade », «Tu voulais Les Mille et Une Nuit tu les as», "Ah, vois notre conteur qui ne conte pas» (Laroui, 2011: 83). Dans le chapitre La rumeur ronfle l'histoire de la vieille femme qui hante le couple des Français dans le raid rue du Hammam prend forme d'une série de contes, car chacun veut en proposer sa propre version. Laroui semble imiter la stratégie de Ben Jelloun qui distribue, à Marrakech, place Jemma-el-Fna, l'histoire d'Ahmed-Zahra à huit conteurs dont le dernier symbolise la parole infinie. Dans les deux romans marrakchis, celui de Ben Jelloun et celui de Laroui, cette stratégie aboutit à des versions contradictoires, chargées de significations multiples. Et finalement la référence à L'Enfant de sable devient presque explicite dans le conte assumé par Cécile: le fils de la vieille dame Tayeb devenu amnésique se transforme à la fin de sa vie en un être de sable : à l'écart du monde, englouti par les vagues de sable, il ne sait plus où finit son corps et où commence la plage et la mer (LAROUI, $2011: 201$ ).

Dans Stéréotype et lecture. Essai sur la réception littéraire, Jean-Louis Dufays - en se référant entre autres à la théorie pragmatique de Michel Picard (Lecture comme jeu) fait la distinction entre les lectures participative (lecteur attentif à l'objet-texte) et intellective (lecteur attentif à l'objet-texte et aux effets qu'il exerce sur lui-même) (DufaYs, 1994: 193-194). Ce clivage est visible dans La vieille dame du riad: le stéréotype oriente aussi bien la lecture participative qu'intellective du roman. Participative, parce que l'histoire prend la source dans le comportement stéréotypé des protagonistes: elle le dénonce en le ridiculisant. Le stéréotype se joue donc sur le plan idéologique du roman. Intellective parce que la structure du texte s'appuie sur le jeu avec des clichés littéraires et avec des formes littéraires figées par la tradition. Et ce jeu nécessite la participation interprétative du lecteur. Les formes discursives schématisées, le recours à des paradigmes romanesques conventionnels 
qui appartiennent à la tradition littéraire française et marocaine, ainsi que des allusions littéraires à un grand degré de lisibilité sont autant de moyens dans le système de persuasion du texte. Fouad Laroui entre en dialogue avec son lecteur (en l'occurrence occidentale), et, en vrai polémiste qui garde surtout le sens de l'humour, utilise comme moyens pour convaincre des clichés, généralisations, schémas narratifs connus. Il combat ainsi des préjugés orientalistes à travers un discours romanesque qui, de manière ostentatoire, ne s'écarte pas des représentations schématiques et formellement «codées» du monde représenté.

\section{Bibliographie}

Amossy, Ruth; Herschberg Pierrot, Anne 1997 : Stéréotypes et clichés. Langue, discours, société. Paris, Nathan.

Ben Jelloun, Tahar 1985 : L'Enfant de sable. Paris, Editions du Seuil.

Borges, Jorge Luis 1985 : Conférences. Paris, Gallimard.

Chelebourg, Christian 2006: Le Surnaturel - Poétique et écriture. Paris, Armand Colin.

DufaYs, Jean-Louis 1994 : Stéréotype et littérature. L'inéluctable va-et-vient. Presse Universitaire de Caen, http://www.openedition.org/6540. Consulté le 29 mai 2019.

Gontard, Marc 1993: Le récit méta-narratif chez Tahar Ben Jelloun. L'intertexte borgésien. In: Tahar Ben Jelloun. Stratégies d'écriture. Dir. Mansour M'Henni. Paris L'Harmattan.

Herschberg Pierrot, Anne 1980: «Problématique du cliché. Sur Flaubert». Poétique, 43, p. 334-345.

Poslaniec, Christian; Houyel, Christine 2001: Activités de lecture à partir de la littérature policière. Paris, Hachette.

Riffaterre, Michel 1979: La Production du texte. Paris, Editions du Seuil, «Poétique».

SAID, Edward W. 2005: L'Orientalisme. L'Orient créé par l'Occident. Paris, Editions du Seuil.

Todorov, Tzvetan 1970: L'Introduction à la littérature fantastique. Paris, Editions du Seuil.

Todorov, Tzvetan 1971 : Poétique de la prose. Paris, Editions du Seuil.

Zekri, Khalid 2006: Fictions du réel. Modernité romanesque et écriture du réel au Maroc 1990-2006. Paris, L'Harmattan.

\section{Note bio-bibliographique}

Magdalena Zdrada-Cok, docteure habilitée à diriger les recherches, professeure de l'Université de Silésie mène ses recherches à l'Institut des Lettres et enseigne dans le Département de Philologie Romane à l'Université de Silésie à Katowice. Elle est spécialiste de littérature postcoloniale francophone et s'intéresse particulièrement aux littératures actuelles du Maghreb, du Machrek 
et de l'Afrique subsaharienne d'expression française. Auteure de deux monographies Tahar Ben Jelloun: hybridité et stratégies de dialogue dans la prose publiée après l'an 2000 (Katowice, 2015) et Les Figures de (Anti-)Narcisse dans l'œuvre de Marguerite Yourcenar (Katowice, 2006) ainsi que de nombreux articles et chapitres d'ouvrage sur, entre autres, T. Ben Jelloun, D. Chraïbi, Abdelkébir Khatibi, Assia Djebar, Leïla Sebbar, K. Daoud, R. Ben Salah, M. Kacimi, A. Sefroui, B. Sansal, A. Rahimi, F. Laroui, A. Kalouaz, M. Yourcenar, M. Tournier, G. Perec, J.M.G. Le Clézio. 